\title{
Accurate Hotspot Localization by Sampling the Near-Field Pattern of Electronic Devices
}

\author{
Prashant Singh, Dirk Deschrijver, Senior Member, IEEE, Davy Pissoort, Member, IEEE, \\ and Tom Dhaene, Senior Member, IEEE
}

\begin{abstract}
This short paper describes a new automated scanning algorithm to identify hotspots (regions with electric or magnetic near-field values above a specific threshold) in the planar near-field profile of electronic systems. The algorithm sequentially determines a set of optimal scanning coordinates where experimental measurements should be performed. The result of the process is a heat map that clearly outlines the presence and localization of hotspots. The efficacy of the proposed algorithm is validated on a measured and a simulated example.
\end{abstract}

Index Terms-Electronic devices, Hotspot detection, Kriging, Near-field scanning, Sequential sampling, Surrogate modeling.

\section{INTRODUCTION}

$\mathbf{T}$ $\mathrm{HE}$ increasing miniaturisation and operating frequencies of electronic devices has led to an elevated risk of intersystem and intrasystem electromagnetic interference (EMI) issues. Early identification of the possible radiating source of such issues is critical for minimizing the design effort and cost while maximizing performance. To this end, electromagnetic near-field (NF) scanning is often used to assess the EMC behaviour of electronic (sub)systems [1]. NF scanning is attractive as it does not require measurements to be taken in (semi)anechoic or reverberant chambers and as it allows the development of equivalent radiation models. Such models can be used to predict the far-field radiation from the NF patterns or they can also be used as component models in commercial electromagnetic software tools [2], [3], [4].

In [5], an automated algorithm was proposed that models the electromagnetic behavior of a device. The aim in [5] was to characterize the overall NF pattern by performing a minimum number of measurements and interpolating the raw NF data into a high-resolution carthography model. However, for EMC pre-compliance testing, one is often interested in identifying only those regions where the near-field values exceed a predefined threshold (i.e., so-called hotspot regions). By focusing solely on those regions of interest, it is possible to significantly reduce the number of measurements and, hence, the overall measurement cost. Therefore, in this letter, a novel scanning algorithm is introduced that is able to detect multiple disjoint hotspot regions by performing batches of measurements in

P. Singh, D. Deschrijver and T. Dhaene are with the Department of Information Technology, iMinds, Ghent University, Ghent 9000, Belgium. (email: [prashant.singh, dirk.deschrijver, tom.dhaene]@ intec.ugent.be)

D. Pissoort is with the Flanders' Mechatronics Engineering Center, Faculty of Engineering Technology, University College Katholieke Hogeschool Brugge-Oostende (KHBO), Ostend, Belgium, and also with ESAT-MICAS at the Katholieke Universiteit Leuven, Leuven 3000, Belgium. (e-mail: davy.pissoort@khbo.be).

Manuscript received April 19, 2005; revised December 27, 2012. a sequential way [6]. In each step, a Kriging model is built and two statistical criteria are used to determine the optimal coordinates where additional measurements are needed. As in [5], the algorithm does not need prior knowledge of the device or the localization of radiation sources.

\section{GOAL STATEMENT AND PRELIMINARIES}

The goal is to build a heat map that localizes all hotspot regions of the device under study while minimizing the number of NF scan points needed. Each scan point, henceforth also called data sample, is represented as a tuple $(\mathbf{x},|F(\mathbf{x})|)$ where $\mathbf{x}$ is a vector that contains the horizontal, vertical and height coordinates of the probe and $|F(\mathbf{x})|$ represents the amplitude of the electric $(E)$ or the magnetic $(H)$ field component. Each spatial coordinate in vector $\mathbf{x}$ is denoted by a superindex $x^{(n)}$.

\section{KRIGING MODELS}

Given a set of $K$ scan points, Kriging interpolation [7] can be used to calculate an analytical model $Y(\mathbf{x})$ of the form

$$
Y(\mathbf{x})=\sum_{p=1}^{P} \beta_{p} f_{p}(\mathbf{x})+\sum_{k=1}^{K} \alpha_{k} \phi\left(\theta, \mathbf{x}_{k}, \mathbf{x}\right),
$$

where coefficients $\beta_{p}$ and $\alpha_{k}$ are estimated from the data by a generalised least squares procedure [5]. The first part corresponds to a regression function through the $K$ scan points with respect to basis function $f_{p}$, while the second part is a localised deviation from the linear regression component. This deviation is a summation of $K$ shifted instances of the Gaussian correlation function, each centered at one scan point

$$
\phi\left(\theta, \mathbf{x}_{k}, \mathbf{x}\right)=\prod_{n=1}^{N} \mathrm{e}^{-\theta_{n}\left|x_{k}^{(n)}-x^{(n)}\right|^{2}}
$$

where $n$ indexes the coordinates of the probe stored in vector $\mathbf{x}$, and the parameters $\theta_{n}$ are identified by the maximum likelihood estimation. More details can be found in [8].

\section{Statistical CRiteria}

The sequential selection of suitable scanning points is critical to identify and localize all hotspots in the given plane. As discussed in [5], they should be chosen in a balanced way to ensure a good mix between exploitation and exploration of the design space. To this end, two statistical criteria are combined, namely the Generalized Probability of Improvement (GPoI) and the minimum distance (MD) criterion. The GPoI criterion takes care of the exploitation part and ensures that the hotspot 
regions are sampled more densely than other areas. The MD criterion takes care of the exploration part and makes sure that the whole design space is properly covered [6].

The algorithm proceeds as follows: first, a set of candidate scan points are randomly generated in the plane according to a uniform distribution, and for each candidate point a score is computed that equals the average score on both criteria (both are normalized to interval $[0,1])$. Then, all the samples are ranked according to this score and the highest ranked samples are picked to form a batch of additional scan points that must be measured. As the NF data from the measurements becomes available, a Kriging model is built that becomes locally more accurate in the regions where hotspots are located while at the same time covering the overall space. This procedure is repeated iteratively until all the hotspots are well described.

More technical details about the two statistical criteria are given in [6] and will be discussed in the following subsections.

\section{A. Generalized Probability of Improvement}

When using Kriging models, the uncertainty at point $\mathrm{x}$ is treated as the realization of a random variable $Y(\mathbf{x})$ with prediction mean $\hat{y}(\mathbf{x})$ and prediction variance $\hat{s}(\mathbf{x})$. The GPoI is used to calculated the probability that the amplitude of the field component $|F(\mathbf{x})|$ at point $\mathbf{x}$ is lying inside a certain range $\left[T_{1}, T_{2}\right]$ :

$$
\begin{aligned}
\operatorname{gPoI}(\mathbf{x}) & =P\left(T_{1} \leq Y(\mathbf{x}) \leq T_{2}\right) \\
& =P\left(Y(\mathbf{x}) \leq T_{2}\right)-P\left(Y(\mathbf{x}) \leq T_{1}\right) \\
& =\int_{-\infty}^{T_{2}} Y(\mathbf{x}) d Y-\int_{-\infty}^{T_{1}} Y(\mathbf{x}) d Y \\
& =\Phi\left(\frac{T_{2}-\hat{y}(\mathbf{x})}{\hat{s}(\mathbf{x})}\right)-\Phi\left(\frac{T_{1}-\hat{y}(\mathbf{x})}{\hat{s}(\mathbf{x})}\right),
\end{aligned}
$$

where $\Phi(t)$ is the standard normal cumulative distribution function. For detection of the hotspot regions, it is proposed to set $T_{1}=\left|F_{\max }\right|-\alpha\left|F_{\max }\right|$ and $T_{2}=\infty$, where $\alpha$ is a desired percentage of the (so far discovered) highest field amplitude $\left|F_{\max }\right|$. Alternatively, one can choose $T_{1}$ as a scalar value to serve as a lower bound that separates hotspot regions from other areas in the plane. By computing the GPoI for various candidate points $\mathbf{x}$, it is possible to determine a new batch of scanning points where hotspots are most likely to be found.

\section{B. Minimum Distance Criterion}

The MD criterion avoids having newly chosen scanning points chosen very close to the existing ones, as they would convey little additional information. Therefore, it calculates the Euclidean distance of a candidate point $\mathrm{x}$ to the closest sample point that has been measured before:

$$
\operatorname{MD}(\mathbf{x})=\gamma \cdot \min _{k=1, \ldots, K} \sqrt{\sum_{n=1}^{N}\left(x^{(n)}-x_{k}^{(n)}\right)^{2}},
$$

where $\gamma$ is a scaling factor which ensures that the output of the MD criterion is normalized to the same range as GPoI.

\section{EXAmPle : MeAsured Microstrip Lines}

As a first example, the algorithm is applied to detect the hotspots of a bent microstrip line that was measured using a NF scanning system. The PCB comprises a $50 \Omega$ microstrip on a $12 \mathrm{~cm} \times 10 \mathrm{~cm}$ FR4 substrate with a thickness of 1.5 $\mathrm{mm}$. To create sufficient radiation, some basic EMC rules were violated on the test PCB (e.g., routing the microstrip over a slot in the ground plane). The microstrip was excited at one end with a generator set at an available power of $0 \mathrm{dBm}$ and terminated in $50 \Omega$ at the other end. The amplitude of each field component $\left|F_{x}\right|$ is measured with a NF scanner that was built from a computer numerical control milling machine. To do this, the miller and its suspension were removed and replaced by a head to which a near-field probe can be attached. The head can be moved automatically in three dimensions above the device under test to perform measurements. The probe is a magnetic NF probe from Langer EMV-Technik (RF-U 2.5-2) that is specified for the frequency range $[30 \mathrm{MHz}-3 \mathrm{GHz}]$. It is connected to a Rohde \& Schwarz EMI receiver and all the measurements are performed at a fixed height of $2 \mathrm{~mm}$ above the printed circuit board (PCB) under test at a frequency of $200 \mathrm{MHz}$ [5]. A layout of the device is shown in Fig. 1.

The goal of the algorithm is to automatically detect all areas in the plane where hotspots are located without assuming prior knowledge of the device. A hotspot can be defined as a region where the amplitude of the values measured with the EMI receiver are bounded within a certain output range $\left[\mathrm{T}_{1}, \mathrm{~T}_{2}\right]$ where the upperbound $T_{2}=\infty$ and lowerbound $T_{1}$ is (for example) set to $35 \mathrm{~dB} \mu \mathrm{V}, 40 \mathrm{~dB} \mu \mathrm{V}$ and $45 \mathrm{~dB} \mu \mathrm{V}$ respectively. As an illustration, the method is here applied to consider the magnetic near field $\left|H_{x}\right|$, although the same procedure can be applied to any field component. First, the algorithm performs an initial set of 100 experiments according to an optimized Latin Hypercube distribution and a Kriging model is built. Then, the optimal location of additional scan points is chosen based on the two statistical criteria (Sect. IV-A and IV-B) and the process of picking additional scan points is repeated in an iterative way. The final results of the measurement process are shown in Figs. 2-4. Scanning points whose output values are within the hotspot range $\left[\mathrm{T}_{1}, \mathrm{~T}_{2}\right]$ are shown as black dots, whereas the other scan points are shown as grey dots. It is clear that the MD criterion ensures that the plane is well covered in such a way that no important regions are missed, whereas GPoI ensures that the scan points are concentrated more densely in hotspots regions.

\section{EXAMPle : Printed Circuit BoARd}

As a second example, a simulated PCB is considered that comprises an $8 \mathrm{~cm} \times 5 \mathrm{~cm}$ FR4 substrate with relative permittivity $=4.2$ and a thickness of $1.5 \mathrm{~mm}$. The bottom layer is a ground plane. On the top layer, there are four traces of various widths, shapes and lengths, which are terminated by different impedances. The top-left corner of the PCB comprises a rectangular dielectric block of $15 \mathrm{~mm} \times 17 \mathrm{~mm}$ and a height of $1 \mathrm{~mm}$, representing, e.g., a package. All details about the traces and the ports are found in Section V of [5]. 


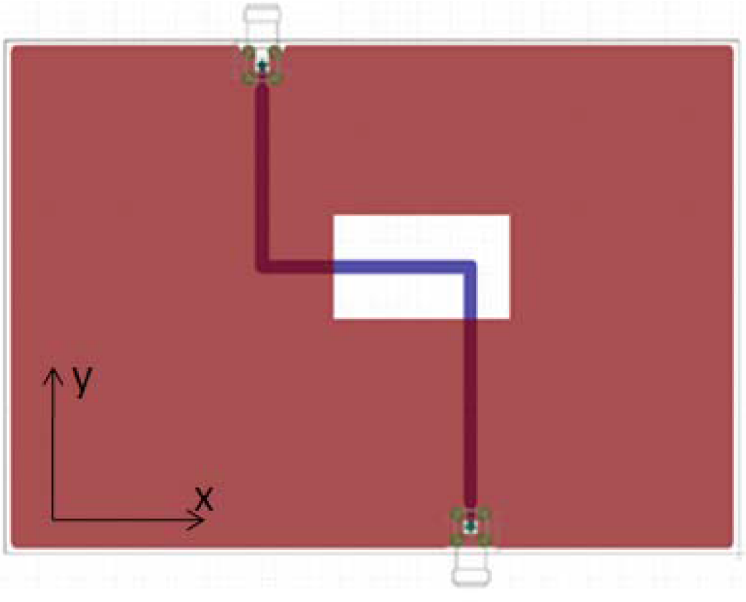

Fig. 1. Layout of the bent microstrip.

In order to facilitate a direct comparison with the results in [5], the same experimental setup will be used. Hence, the amplitude of magnetic field component $\left|H_{x}\right|$ is simulated with Agilent's EMpro at a fixed height of $2 \mathrm{~mm}$ above the PCB over an area of $10 \mathrm{~cm} \times 7 \mathrm{~cm}$, and this at a frequency of $900 \mathrm{MHz}$. Rather than modeling the field component over the entire plane as in [5], the novel algorithm performs only as few measurements as are needed to detect the hotspot areas. As an example, a hotspot is here defined as a region where the NF values are larger than $\alpha=80 \%$ of the highest field amplitude. The algorithm was applied to sequentially perform simulations until all hotspots are discovered. The 300 selected scan points are shown in Fig. 5 and a heat map of the corresponding Kriging model is shown in Fig. 6. It is clear that the algorithm concentrates scan points in regions where the NF values are higher than the specified threshold (red dots), while exploring also the other regions of the plane (blue dots).

In order to validate the results, the "true" NF pattern was also calculated by performing a large amount of simulations on a uniform grid of 7171 scan points. The NF pattern (which is unknown to the algorithm) is shown in Fig. 7 and the hotspot threshold is marked as a horizontal slice. By comparing Figs. $5-7$, it is confirmed that the algorithm is indeed able to discover all hotspot regions by performing only $300 \mathrm{NF}$ scans.

If one builds a full carthography model, as was the aim in [5], then 700 scan points would be needed to have a globally accurate model (i.e. a heat map that accurately captures all the variations in the NF pattern). Using the new approach that focuses only on the localization and identification of hotspot regions, the number of scan points can be reduced even further to 300 , which leads to significant savings in measurement cost.

\section{CONCLUSION}

A novel NF scanning algorithm that detects and localizes hotspot regions is proposed for EMC compliance testing of electronic devices. The algorithm sequentially performs a limited set of NF scans in the plane and evaluates two statistical criteria to determine the optimal coordinates where additional measurements should be performed. The outcome of the process is a heat map that clearly visualizes the presence and

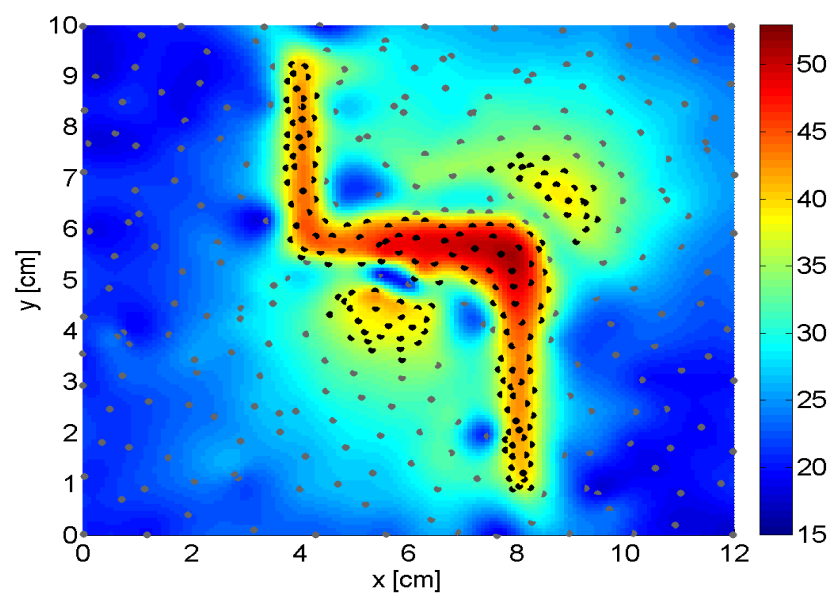

Fig. 2. Bent microstrip : hotspot defined as $[35 \mathrm{~dB} \mu \mathrm{V}-\infty]$.

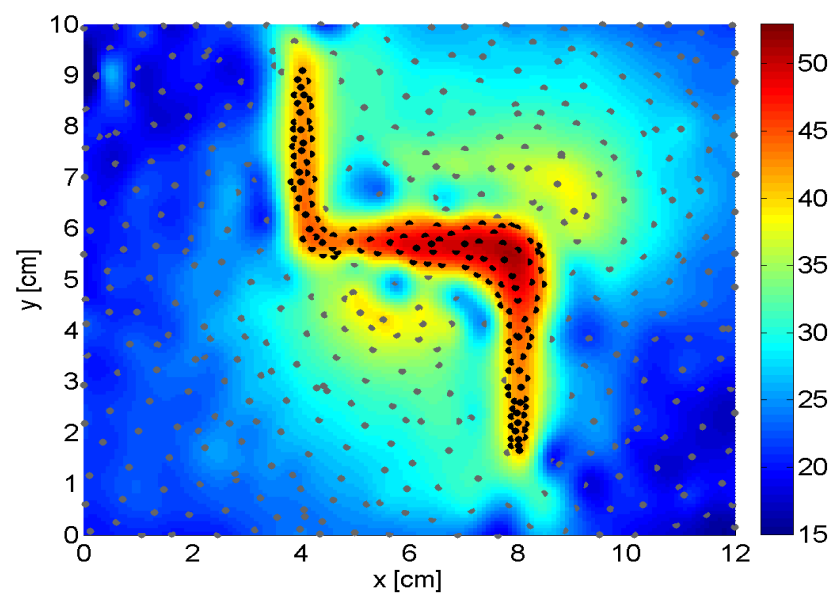

Fig. 3. Bent microstrip : hotspot defined as $[40 \mathrm{~dB} \mu \mathrm{V}-\infty]$.

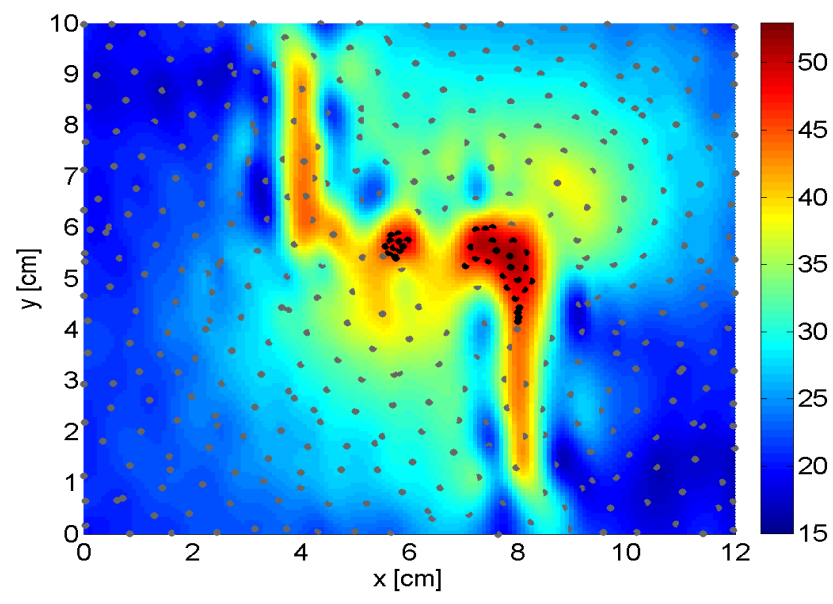

Fig. 4. Bent microstrip : hotspot defined as $[45 \mathrm{~dB} \mu \mathrm{V}-\infty]$.

the localization of hotspot regions. It is found that this method may require significantly less scan points when compared to a dense uniform sampling, or other approaches which aim at a globally accurate carthography of the NF pattern [5]. The effectiveness of the algorithm is demonstrated by applying it to a measured microstrip example and a simulated PCB. 


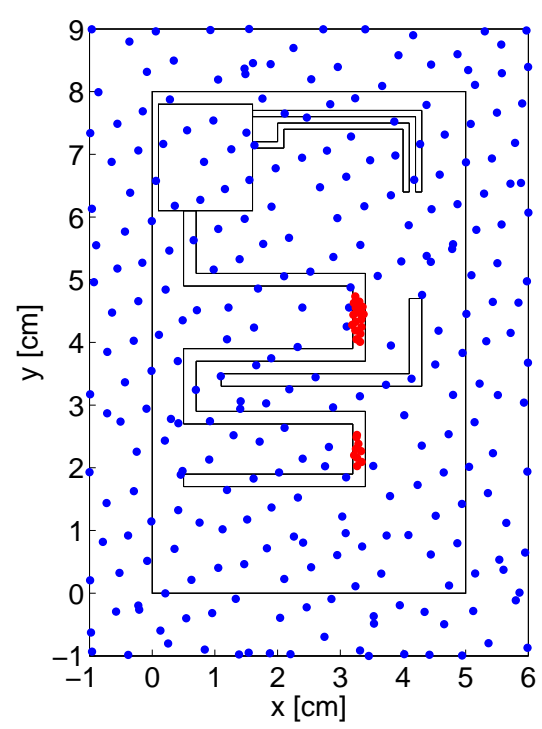

Fig. 5. PCB example : distribution of the 300 scan points.

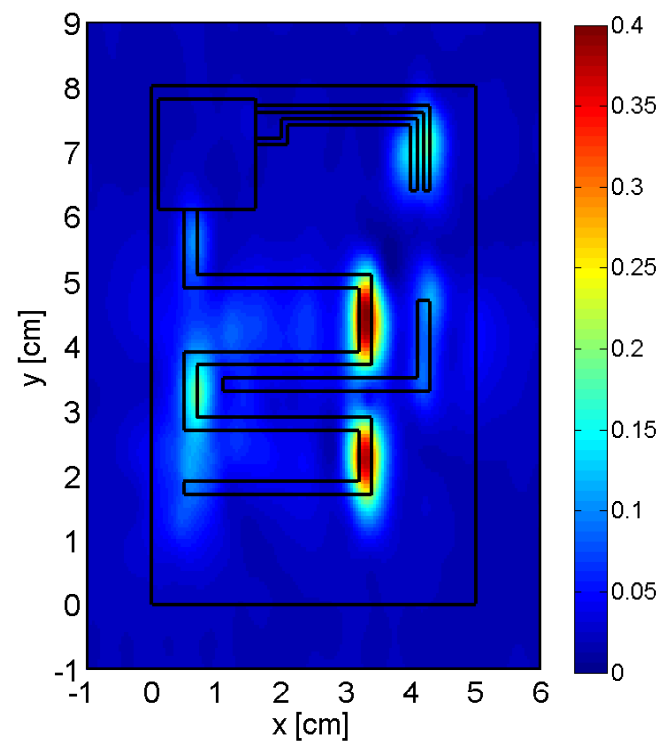

Fig. 6. PCB example : heat map of Kriging model.

\section{SOFTWARE IMPLEMENTATION}

All experiments were performed using the SUMO research platform [9] which is freely available for non-commercial, personal and academic use at http://sumo.intec.ugent.be.

\section{ACKNOWLEDGMENT}

This research has been funded by the IWT (Flanders, Belgium) through the Technology Transfer project NEATH, by the Interuniversity Attraction Poles Programme BESTCOM initiated by the Belgian Science Policy Office, and the Research Foundation Flanders (FWO-Vlaanderen). Dirk Deschrijver is a post-doctoral researcher of FWO-Vlaanderen.

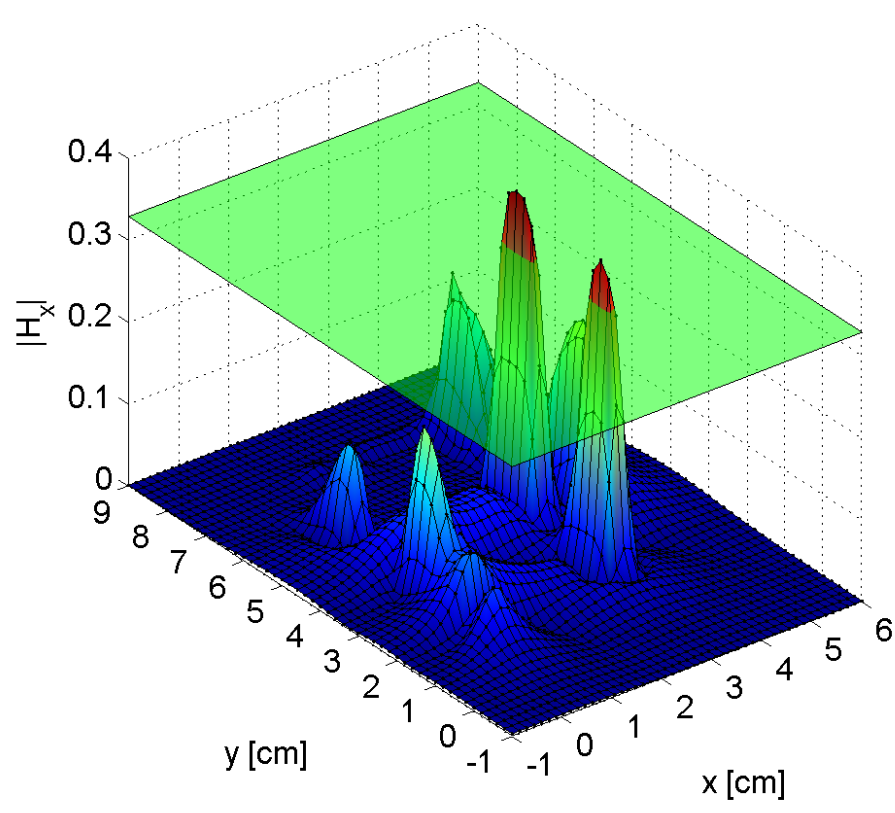

Fig. 7. PCB example : NF pattern based on a uniform grid of 7171 simulations

\section{REFERENCES}

[1] D. Baudry, C. Arcambal, A. Louis, B. Mazari, and P. Eudeline, "Applications of the near-field techniques in emc investigations," Electromagnetic Compatibility, IEEE Transactions on, vol. 49, no. 3, pp. 485 -493, Aug. 2007.

[2] Y. Alvarez, M. Rodriguez, F. Las-Heras, and M. Hernando, "On the use of the source reconstruction method for estimating radiated EMI in electronic circuits," Instrumentation and Measurements, IEEE Transactions on, vol. 59, no. 12, pp. 3174-3183, 2010.

[3] P. Fernandez Lopez, D. Baudry, V. S, and B. Mazari, "Simple electromagnetic modeling procedure: From near-field measurements to commercial electromagnetic simulation tool," Instrumentation and Measurements, IEEE Transactions on, vol. 59, no. 12, pp. 3111-3121, 2010.

[4] H. Weng, D. Beetner, and R. DuBroff, "Applications of the near-field techniques in emc investigations," Electromagnetic Compatibility, IEEE Transactions on, vol. 53, no. 4, pp. 891-899, 2011.

[5] D. Deschrijver, F. Vanhee, D. Pissoort, and T. Dhaene, "Automated nearfield scanning algorithm for the emc analysis of electronic devices," Electromagnetic Compatibility, IEEE Transactions on, vol. 54, no. 3, pp. $502-510$, Jun. 2012

[6] I. Couckuyt, J. Aernouts, D. Deschrijver, F. Turck, and T. Dhaene, "Identification of quasi-optimal regions in the design space using surrogate modeling," Engineering with Computers, vol. 29, no. 2, pp. 127-138, Apr. 2012.

[7] J. Sacks, W. J. Welch, T. J. Mitchell, and H. P. Wynn, "Design and analysis of computer experiments," Statistical science, vol. 4, no. 4, pp. 409-423, 1989.

[8] I. Couckuyt, F. Declercq, T. Dhaene, H. Rogier, and L. Knockaert, "Surrogate-based infill optimization applied to electromagnetic problems," International Journal of $R F$ and Microwave Computer-Aided Engineering, vol. 20, no. 5, pp. 492-501, 2010.

[9] D. Gorissen, I. Couckuyt, P. Demeester, T. Dhaene, and K. Crombecq, "A surrogate modeling and adaptive sampling toolbox for computer based design," The Journal of Machine Learning Research, vol. 11, pp. 20512055, 2010. 